\title{
Clustering and Uncertainty in Perfect Chaos Systems
}

\author{
Sergey A. Kamenshchikov \\ Faculty of Physics, Moscow State University of M. V. Lomonosov, Russian Federation, Leninskie Gory, Moscow 119991, Russia \\ Correspondence should be addressed to Sergey A. Kamenshchikov; kamphys@gmail.com
}

Received 26 November 2013; Revised 9 February 2014; Accepted 26 February 2014; Published 26 March 2014

Academic Editor: Grzegorz Litak

Copyright (C) 2014 Sergey A. Kamenshchikov. This is an open access article distributed under the Creative Commons Attribution License, which permits unrestricted use, distribution, and reproduction in any medium, provided the original work is properly cited.

\begin{abstract}
The goal of this investigation was to derive strictly new properties of chaotic systems and their mutual relations. The generalized Fokker-Planck equation with a nonstationary diffusion has been derived and used for chaos analysis. An anomalous transport turned out to be natural property of this equation. A nonlinear dispersion of the considered motion allowed us to find a principal consequence: a chaotic system with uniform dynamic properties tends to instable clustering. Small fluctuations of particles density increase by time and form attractors and stochastic islands even if the initial transport properties have uniform distribution. It was shown that an instability of phase trajectories leads to the nonlinear dispersion law and consequently to a space instability. A fixed boundary system was considered, using a standard Fokker-Planck equation. We have derived that such a type of dynamic systems has a discrete diffusive and energy spectra. It was shown that phase space diffusion is the only parameter that defines a dynamic accuracy in this case. The uncertainty relations have been obtained for conjugate phase space variables with account of transport properties. Given results can be used in the area of chaotic systems modelling and turbulence investigation.
\end{abstract}

\section{Introduction}

Several scenarios of a turbulence transition have been proposed since 1883 when the turbulence concept was firstly introduced by an English engineer called Osborne Reynolds. The dynamic phase transition in a liquid stream was remarked by Reynolds-it was characterized by an unstable vortex appearance and two limit states of motion: laminar and turbulent types. Since an introduction of a turbulence concept, its properties have been generalized and transformed into properties of a chaos state.

Several scenarios of a turbulence transition have been developed. Among them, the Landau-Hopf instability mechanism [1], the Lorenz attractor mechanism [2], the scenario of Poincare-Feigenbaum [3], and Kolmogorov-ArnoldMoser [4]. Each of the outlined mechanisms has its basic assumptions and an individual area of its application. For this reason none of them suggests some universal approachesmoreover unambiguous connections between the given mechanisms are not stated yet. Let us try to highlight common points for the formulation of chaos concept.

Consent has been obtained that an unpredictability of chaos is consequence of two conditions: a finite resolution of generalized phase space and instability of phase trajectories and mixing of phase trajectories [5]. Formally these conditions can be defined in the following ways (1), (2), and (3):

$$
{ }_{\Delta} x^{i} \geq{ }_{\Delta} x_{\min }^{i}>0 .
$$

Here ${ }_{\Delta} x_{\min }^{i}$ is an element of the Hilbert phase space while $\vec{x}(t)$ is the characteristic vector; each dynamic state corresponds to a given $\vec{x}$. For the case of independent agents, a dimension of the phase space is defined according to a number of agents and Euclidean dimension.

A second condition can be expressed through the sum of positive Lyapunov factors $\sigma_{i}^{+}$for each dimension of phase space:

$$
\sum_{i=1}^{P} \sigma_{i}^{+}(\vec{x})>0 .
$$

Here $P$ corresponds to a number of phase space dimensions. A realization of these chaos conditions leads to another basic consequence. Under the condition of a phase space mixing reverse mapping for time $t(\vec{x})$ is not single valued in general case. Mixing property can be expressed through the 
autocorrelation function $R(f(\vec{x}), \tau)$, where $f$ is an arbitrary dynamic function (e.g., momentum):

$$
\lim _{\tau \rightarrow \infty} R(f(\vec{x}), \tau)=0 .
$$

Here $\tau$ is a delay time between the start and the end of a system evolution observation. Condition (3) is naturally satisfied for random walks. A realization of this condition automatically leads to an execution of the Slutsky criterion for an ergodic system:

$$
\lim _{\substack{T \rightarrow \infty \\ \tau \rightarrow \infty}} \int_{0}^{T} R(f(\vec{x}), \tau) \cdot\left(1-\frac{\tau}{T}\right) d \tau=0 .
$$

According to (4), system becomes ergodic for $\tau \rightarrow \infty$. For real physical systems ergodicity corresponds to the following relation:

$$
\begin{aligned}
& { }_{\Delta} t_{\min }>{ }_{\Delta} t_{\text {ins }}, \quad{ }_{\Delta} t_{\text {ins }}=\frac{1}{h}=\frac{1}{\sum_{i=1}^{K} \sigma_{i}^{+}(\vec{x})}, \\
& \langle h\rangle=\bar{h}=h_{d} .
\end{aligned}
$$

Here ${ }_{\Delta} t_{\min }$ is an available time resolution and ${ }_{\Delta} t_{\text {ins }}$ is the instability increment expressed through the integrated Lyapunov factors. Andrey Kolmogorov has shown that an averaged $h$ corresponds to the Gibbs entropy production:

$$
h_{d}=\frac{\partial S}{\partial t}=\frac{\partial}{\partial t} \ln (\Gamma) \text {. }
$$

In such a way positive $h$ necessarily leads to the positive dynamic entropy. Finally we may define complex portrait of chaotic system as a complex dynamic system that corresponds to given obligatory properties: a finite resolution of phase space, instability of phase trajectories, mixing of phase trajectories, and a positive entropy production. In the next sections we will discuss relative and nonlinear properties of chaos in detail. However all properties that have been shown above are assumed to be satisfied.

\section{Nonlinear Dispersion and Clustering}

Let us consider one of the possible approaches to the description of chaos which is the partial differential equation of Fokker-Planck. This model has been modified and finalized by Kolmogorov in 1938 [5]. The basis used for its formulation is Chapman-Kolmogorov equation. Let us consider a onedimensional case (for simplicity); then a transitional probability for random walks $x_{1}, t_{1} \rightarrow x_{3}, t_{3}$ formally satisfies the following Markovian relation:

$$
\begin{aligned}
W & \left(x_{3}, t_{3} \mid x_{1}, t_{1}\right) \\
\quad & =\int d x_{2} W\left(x_{3}, t_{3} \mid x_{2}, t_{2}\right) \cdot W\left(x_{2}, t_{2} \mid x_{1}, t_{1}\right) .
\end{aligned}
$$

In the above formula $x(t)$ is a system coordinate, while $W\left(x, t \mid x_{0}, t_{0}\right)$ is a probability density of $x(t)$; location subject to the initial coordinate is $x_{0}\left(t_{0}\right)$. Fokker-Planck-Kolmogorov (FPK) equation has been received based on the following assumptions.
(A1) $W\left(x^{\prime}, t^{\prime} \mid x, t\right)=W\left(x^{\prime}, x, t^{\prime}-t\right)=W\left(x^{\prime}, x,{ }_{\Delta} t\right)$. A transitional probability does not depend on the initial time point. This demand implies that condition of $\tau \gg>\tau_{C}$ is satisfied, where $\tau_{C}$ is an effective width of autocorrelation function for $x(t)$.

(A2) $P\left(x^{\prime}, t\right)=W\left(x^{\prime}, x, t\right)$. A final probability does not depend on the initial coordinate. This restriction implies $\tau>>\tau_{C}$ as well.

(A3) The initial distribution density is defined by Dirac delta function: $W(x)=\delta(0)$; we assume that the initial coordinate can be defined quite accurately in relation to the general size of a considered system.

(A4) According to (A3) assumption, an approximation of the second order may be received for the transitional probability:

$$
\begin{aligned}
W\left(x, x_{0},{ }_{\Delta} t\right)= & \delta\left(x-x_{0}\right)+a\left(x,{ }_{\Delta} t\right) \cdot \delta^{\prime}\left(x-x_{0}\right) \\
& +\frac{1}{2} \cdot b\left(x,{ }_{\Delta} t\right) \cdot \delta^{\prime \prime}\left(x-x_{0}\right) .
\end{aligned}
$$

Factors $a\left(x,{ }_{\Delta} t\right)$ and $b\left(x,{ }_{\Delta} t\right)$ are defined by relations (8) and (9):

$$
\begin{aligned}
& a\left(x,{ }_{\Delta} t\right)=\int\left(x-x_{0}\right) \cdot W\left(x, x_{0},{ }_{\Delta} t\right) d x_{0}=\left\langle\left\langle{ }_{\Delta} x\right\rangle\right\rangle, \\
& b\left(x,{ }_{\Delta} t\right)=\int\left(x-x_{0}\right)^{2} \cdot W\left(x, x_{0},{ }_{\Delta} t\right) d x_{0}=\left\langle\left\langle{ }_{\Delta} x^{2}\right\rangle\right\rangle .
\end{aligned}
$$

A substitution of expansion (8) into Chapman-Kolmogorov equation [6] gives us the following form for $P\left(x^{\prime}, t\right)=$ $W\left(x^{\prime}, x, t\right)$ standard Fokker-Planck equation:

$$
\begin{gathered}
\frac{\partial P(x, t)}{\partial t}=\frac{1}{2} \cdot \frac{\partial}{\partial x}\left(B(x) \cdot \frac{\partial P(x, t)}{\partial x}\right), \\
B(x)=\lim _{\Delta_{t \rightarrow 0}}\left(\frac{\left\langle\left\langle{ }_{\Delta} x^{2}\right\rangle\right\rangle}{{ }_{\Delta} t}\right)=\frac{\left\langle\left\langle{ }_{\Delta} x^{2}\right\rangle\right\rangle}{{ }_{\Delta} t_{\min }} .
\end{gathered}
$$

Now we may extend this model to the phase systems with phase mixing. Let us define a specific energy of a complex system:

$$
\varepsilon(x, t)=\lim _{\Delta^{t \rightarrow 0}}\left(\frac{\Delta^{x}(x, t)}{{ }_{\Delta} t}\right)^{2} \approx\left(\frac{\Delta^{x}(x, t)}{{ }_{\Delta} t_{\min }}\right)^{2} .
$$

A second transport factor can be expressed then through this quantity in the following way:

$$
\begin{aligned}
B(x, t) & \approx \frac{\left\langle\left\langle{ }_{\Delta} x^{2}\right\rangle\right\rangle}{{ }_{\Delta} t_{\min }} \\
& ={ }_{\Delta} t_{\min } \cdot \int \varepsilon(x, t) \cdot W\left(x, x_{0},{ }_{\Delta} t\right) d x_{0} \\
& ={ }_{\Delta} t_{\min } \cdot\langle\langle\varepsilon(x, t)\rangle\rangle .
\end{aligned}
$$


For a conservative case an averaged specific energy is presented as $\langle\langle\varepsilon(x, t)\rangle\rangle=\langle\langle\varepsilon(x)\rangle\rangle$. According to relation (11) this means that transport factor does not depend on time explicitly and standard FPK is valid. However this expression is not satisfied if $x(t) \leftrightarrow \varepsilon(t)$ mutual correspondence is absent. According to (13) the averaged specific energy conservancy may be violated in this case. A mutual correspondence is distorted because a phase trajectory mixing occurs; several system states are possible for the same phase space location. A second transport factor $B(x)$ in fact expresses diffusion in phase space, as it was remarked by Zaslavsky [6]. For the considered type of motion it has explicit time dependence: $B(x, t)$. According to the introduced division we will consider two types of phase space behavior. First one corresponds to $\langle\langle\varepsilon(x, t)\rangle\rangle=\langle\langle\varepsilon(x)\rangle\rangle$ and can be designated as phase conservative state. Then the second one $\langle\langle\varepsilon(x, t)\rangle\rangle$ relates to a phase mixing; we will call it transitional state. It is more general and includes phase state as the particular case.

Taking into account the nonstationary properties we may modify the assumption (A4) in the following way:

$$
\begin{aligned}
W\left(x, x_{0},{ }_{\Delta} t\right)= & \delta\left(x-x_{0}\right)+a\left(x,{ }_{\Delta} t, t\right) \cdot \delta^{\prime}\left(x-x_{0}\right) \\
& +\frac{1}{2} \cdot B\left(x,{ }_{\Delta} t, t\right) \cdot \delta^{\prime \prime}\left(x-x_{0}\right) .
\end{aligned}
$$

Derivative of a given probability $P\left(x^{\prime}, t\right)$ is represented according to the Chapman-Kolmogorov equation in the usual form:

$$
\begin{aligned}
& \frac{\partial P(x, t)}{\partial t} \\
& =\lim _{\Delta^{t} \rightarrow 0}\left[\frac { 1 } { { } _ { \Delta } t } \cdot \left(\int d x \cdot P\left(x_{0}, t\right)\right.\right. \\
& \left.\left.\quad \cdot\left(W\left(x, x_{0},{ }_{\Delta} t\right)-\delta\left(x-x_{0}\right)\right)\right)\right] .
\end{aligned}
$$

Substitution of (14) into (15) gives nonconservative FPK:

$$
\frac{\partial P(x, t)}{\partial t}=\frac{1}{2} \cdot \frac{\partial}{\partial x}\left(B(x, t) \cdot \frac{\partial P(x, t)}{\partial x}\right) .
$$

In the given relation, $D(x, t)=\sqrt{2 \cdot B(x, t)}$ corresponds to an anomalous diffusion factor. We have extended the approach of Kolmogorov to an arbitrary class of systems that takes into account obligatory mixing properties.

Let us consider the simplest case of the uniform diffusion: $D(x, t)=D(t)$. This problem in fact relates to the simplest transitional case. It can be used as first approximation of chaotic description as well. A Fourier decomposition of (16) then gives us the following relation:

$$
\begin{aligned}
& \int i \omega \cdot P_{\omega}(x, \omega) \cdot \exp (-i \omega \cdot t) d \omega \\
& \quad=-\int B(t) \cdot(k)^{2} P_{k}(k, t) \cdot \exp (-i k \cdot x) d k .
\end{aligned}
$$

Here $B(t) \rightarrow B(t) / 2$ is a modified transport factor. Second decomposition gives relations (18) with Fourier operator's kernel $\widehat{P}(k, \omega)$ :

$$
\begin{gathered}
P_{k}(k, t)=\frac{1}{\sqrt{2 \pi}} \int_{-\Omega}^{\Omega} \widehat{P}(k, \omega) \cdot \exp (-i \omega \cdot t) d \omega, \\
P_{\omega}(x, \omega)=\frac{1}{\sqrt{2 \pi}} \int_{-K}^{K} \widehat{P}(k, \omega) \cdot \exp \left(-i k_{j} \cdot x_{j}\right) d k_{j} .
\end{gathered}
$$

Here spectral amplitudes are finite according to the normalization condition for the probability. Integrals limits are defined according to the Kotelnikov theorem: $\Omega=$ $\left(2 \cdot{ }_{\Delta} t_{\min }\right)^{-1}, K=\left(2 \cdot{ }_{\Delta} x_{\min }\right)^{-1}$. Substitution of (18) into (17) gives wave packet form of FPK:

$$
\begin{aligned}
& \int_{-\Omega}^{\Omega} \int_{-K}^{K} i \omega \cdot \widehat{P}(k, \omega) \cdot \exp (-i \omega t-i k x) d k_{j} d \omega \\
& =-\int_{-\Omega}^{\Omega} \int_{-K}^{K} B(t) \cdot k^{2} \cdot \widehat{P}(k, \omega) \cdot \exp (-i \omega t-i k x) d k_{j} d \omega .
\end{aligned}
$$

A general arbitrariness of integration limits allows representing of $\omega\left(k_{i}\right)$ dispersion law:

$$
\omega(k)=i \cdot B(t) \cdot(k)^{2} .
$$

As it follows from outlined expression nonlinear dispersion law of (20) is mandatory property of uniform chaotic state. Allocation of a real part leads to the relation

$$
\operatorname{Im}(k)=-B(t) \cdot \frac{\operatorname{Re}(k)}{\operatorname{Re}(\omega)}, \quad \operatorname{Im}(k) \leq 0 .
$$

A first Fourier decomposition of the probability density then can be given by the following equation:

$$
\begin{aligned}
& P(x, t) \\
& \quad=\int P_{k}(k, t) \cdot \exp (|\operatorname{Im}(k)| \cdot x) \exp (-i \operatorname{Re}(k) \cdot x) d k .
\end{aligned}
$$

The imaginary part of (20) corresponds to the relation

$$
\operatorname{Im}(\omega)=B(t) \cdot|k|^{2}=\alpha(t), \quad \alpha(t)>0 .
$$

A first Fourier decomposition of the probability density can be expressed then in the following way:

$$
\begin{aligned}
P & (x, t) \\
& =\int P_{\omega}(x, \omega) \cdot \exp (|\operatorname{Im}(\omega)| \cdot t) \exp (-i \operatorname{Re}(\omega) \cdot t) d \omega .
\end{aligned}
$$

According to the relations (22) and (24), a chaotic system with uniform dynamic properties defined by factor $D(t)$ tends to instable clustering; small fluctuations of particles density increase by time and form attractors, repellers, and stochastic 
islands. This process leads to destruction of dynamic uniformity and finally this approximation becomes incorrect; we need to solve a general form of extended FPK (16). However it is important to notice that clustering is a natural property of chaotic systems; it relates to the first approximation of any transition. In fact we have shown how instability of phase trajectories leads to the nonlinear dispersion (20) and consequently to a space instability.

\section{Uncertainty Relations}

In the previous section we have proved logically that clustering is a sufficient property of the simplest transitional state. In this section we will consider a trivial case, a phase state with a fixed boundary. However, nontrivial uncertainty relations will be derived based on a standard one-dimensional FokkerPlanck equation.

Let us consider a conservative problem of the fixed boundary:

$$
\begin{gathered}
\frac{\partial P(x, t)}{\partial t}=\frac{B}{2} \cdot \frac{\partial^{2} P(x, t)}{\partial x^{2}}, \\
P(0, t)=P(L, t)=0 \quad x \in[0, L], \\
P(x, 0)=P_{0}(x) .
\end{gathered}
$$

Equation (25) mathematically corresponds to a uniform linear diffusion PDE. Here $B=$ const according to the condition of an energy conservancy; we consider the system's equilibrium phase state:

$$
\begin{aligned}
B(x, t) & \approx{ }_{\Delta} t_{\min } \cdot \varepsilon \cdot \int_{0}^{L} W\left(x, x_{0},{ }_{\Delta} t\right) d x_{0} \\
& =\varepsilon \cdot{ }_{\Delta} t_{\min }=\text { const. }
\end{aligned}
$$

A solution of (25) may be searched in a form of the Fourier expansion

$$
\begin{gathered}
P(x, t)=\sum_{j=1}^{N} c_{j}(t) \cdot \sin \left[\left(\frac{\pi \cdot j}{L}\right) \cdot x\right], \\
c_{j}(t)=\frac{2}{L_{x}} \cdot \int_{0}^{L} P(\zeta, t) \cdot \sin \left[\left(\frac{\pi \cdot j}{L}\right) \cdot \xi\right] d \xi .
\end{gathered}
$$

A substitution of (27) into (25) gives the following superposition of modes:

$$
\sum_{j=1}^{N} \sin \left[\left(\frac{\pi \cdot j}{L}\right) \cdot x\right] \cdot\left(\frac{\partial c_{j}(t)}{\partial t}+\frac{B \cdot c_{j}(t)}{2} \cdot\left(\frac{\pi \cdot j}{L}\right)^{2}\right)=0 .
$$
value:

For an arbitrary $L$, a second factor has an obligatorily zero

$$
\frac{\partial c_{j}(t)}{\partial t}=-\frac{B \cdot c_{j}(t)}{2} \cdot\left(\frac{\pi \cdot j}{L}\right)^{2}
$$

Then, possible values of a transport factor $B$ may be expressed in the following way:

$$
B_{j}=-\frac{2}{c_{j}(t)} \cdot \frac{d c_{j}(t)}{d t} \cdot\left(\frac{L}{\pi \cdot j}\right)^{2} .
$$

Let us consider the instability of the particles density: $c_{j}(t)=c_{j}(0) \cdot \exp \left(\phi_{j} \cdot t\right)$. It leads to a clustering of particles, considered in the previous section, and finally to the nonstationary regime when $B=B(t)$.

Here $c_{j}^{0}=c_{j}(0)$ corresponds to an initial density probability, defined by the third relation in group (25). Factors $\phi_{j}$ characterize modes instability increments and depend on nonlinear properties of the complex system media. They are assumed to be constant in the given approximation for the given complex system. Here we consider the states in the vicinity of a conservative condition. A normalization condition for $P(x, t)$ can be represented in the following way:

$$
\sum_{j=1}^{N} c_{j}^{0} \cdot \exp \left(\phi_{j} \cdot t\right) \int_{0}^{L} \cdot \sin \left[\left(\frac{\pi \cdot j}{L}\right) \cdot x\right] d x=1
$$

We can remark that satisfaction of this condition is possible only if both relations $\phi_{j}>0$ and $\phi_{j} \prec 0$ are valid for the different modes of the spectrum. It means that increasing and decreasing of quasiregular fluctuations of particles density occur in the media of a complex system.

According to the relation (30) we have discrete spectrum of phases that can be realized for this considered case:

$$
\begin{array}{r}
B_{j}=2 \cdot \phi_{j} \cdot\left(\frac{L}{\pi \cdot j}\right)^{2}, \quad \varepsilon_{j}=\frac{2 \cdot \phi_{j}}{{ }_{\Delta} t_{\min }} \cdot\left(\frac{L}{\pi \cdot j}\right)^{2}, \\
j=\overline{1, N .}
\end{array}
$$

Let us introduce a circular frequency $\omega=2 \pi /{ }_{\Delta} t_{\min }$. Then the relation (32) can be modified:

$$
\Delta \varepsilon_{j}=\frac{\phi_{j}}{\pi^{3}} \cdot\left(\frac{L}{j}\right)^{2} \cdot \Delta \omega .
$$

It is known that a perfect accuracy substitution for the initial coordinate of particle can be represented by Dirac function:

$$
P\left(x\left(t_{0}\right)\right)=\delta\left(x(t)-x_{j}\left(t_{0}\right)\right)
$$

Normalization condition for $P\left(x_{j}\left(t_{0}\right)\right)$ then can be represented in the following way:

$$
\int_{0}^{L} \delta\left(x(t)-x\left(t_{0}\right)\right) d x_{j}=\int_{0}^{L_{j}}\left(\sum_{k} \frac{\delta\left(t-t_{0}\right)}{\left|\partial x_{j}^{k}(t) / \partial t\right|}\right) d x
$$

Dirac functional is represented here through the time argument.

Index $k$ corresponds to zeros of function $x(t)$. In considered case we have only one value of argument, corresponding 
to zero $t_{0}$. Then relation (35) can be modified in the following way:

$$
\begin{gathered}
\lim _{t \rightarrow t_{0}}\left[\int_{0}^{L}\left(\sum_{k} \frac{\delta\left(t-t_{0}\right)}{\left|\partial x^{k}(t) / \partial t\right|}\right) d x\right] \\
=\frac{\partial x(t) / \partial t}{|\partial x(t) / \partial t|} \int_{0}^{T(L)} \delta\left(t-t_{0}\right) d t .
\end{gathered}
$$

As we can see in a vicinity of $t_{0}\left(t \rightarrow t_{0}, x_{j} \rightarrow\right.$ $\left.x_{j}\left(t_{0}\right)\right)$ space-time correspondence allows introducing of a probability density:

$$
\delta\left(x(t)-x\left(t_{0}\right)\right) \longleftrightarrow \delta\left(t-t_{0}\right) \cdot \operatorname{sign}\left(\frac{\partial x(t)}{\partial t}\right) .
$$

Real physical systems correspond to a finite space and time resolution: ${ }_{\Delta} t \rightarrow 0$ and ${ }_{\Delta} x \rightarrow 0$ in fact correspond to ${ }_{\Delta} t=$ $2 \cdot{ }_{\Delta} t_{\min }$ and ${ }_{\Delta} x=2 \cdot{ }_{\Delta} x_{\min }$.

Limitations of our observance qualities lead to a discrete alternative of Delta function:

$$
\begin{aligned}
& x(t)= \begin{cases}C_{1} & x<\left|{ }_{\Delta} x_{\min }\right| \\
0 & x \geq\left|{ }_{\Delta} x_{\min }\right|,\end{cases} \\
& t\left(x_{j}\right)= \begin{cases}C_{2} & \left.t \prec\right|_{\Delta} t_{\min } \mid \\
0 & t \geq\left.\right|_{\Delta} t_{\min } \mid .\end{cases}
\end{aligned}
$$

Here we assume without a loss of generality that $t_{0}=0$. According to a normalization condition for the probability, coefficients $C_{1}$ and $C_{2}$ can be expressed in the following way:

$$
C_{1}=\frac{1}{2 \cdot{ }_{\Delta} x_{\min }}, \quad C_{2}=\frac{1}{2 \cdot{ }_{\Delta} t_{\min }} .
$$

Relations (38) correspond to a rectangular function. Its Fourier spectrum is expressed through

$$
\begin{gathered}
S_{x}\left(\omega_{x}\right)=C_{1} \cdot{ }_{\Delta} x_{\min } \cdot \operatorname{Sinc}\left(\frac{\omega_{x} \cdot{ }_{\Delta} x_{\min }}{2}\right), \\
S_{t}\left(\omega_{t}\right)=C_{2} \cdot{ }_{\Delta} t_{\min } \cdot \operatorname{Sinc}\left(\frac{\omega_{t} \cdot{ }_{\Delta} t_{\min }}{2}\right) .
\end{gathered}
$$

A central maximum of the second Sinc function is limited by the interval ${ }_{\Delta} \omega_{t}=2 \pi /{ }_{\Delta} t_{\min }$ between two first zeroes. This interval includes the biggest part of Sinc function integral value. In such a way ${ }_{\Delta} \omega_{t}=2 \pi /{ }_{\Delta} t_{\min }$ can be used as a characteristic width of the probability spectrum. In the same way we obtain a space frequency width:

$$
{ }_{\Delta} \omega_{t} \geq \frac{2 \pi}{{ }_{\Delta} t_{\min }}, \quad{ }_{\Delta} \omega_{x} \geq \frac{2 \pi}{{ }_{\Delta} x_{\min }} .
$$

A substitution of the first relation into (33) gives us the following expression:

$$
{ }_{\Delta} \varepsilon_{j} \cdot{ }_{\Delta} t_{\min } \geq \frac{2 \phi_{j}}{\left(k_{j}\right)^{2}} .
$$

Here the wave number $k_{j}=(\pi \cdot j) / L$ is introduced. If we express an increment $\phi_{j}$ through the diffusion factor and according to (32) we may derive a final uncertainty relation:

$$
{ }_{\Delta} \varepsilon_{j} \cdot{ }_{\Delta} t_{\min } \geq B_{j} .
$$

A minimal spectral diffusion $B_{0}=\left(B_{j}\right)_{\min }$ allows us to simplify this equation in the following way:

$$
{ }_{\Delta} \varepsilon_{j} \cdot{ }_{\Delta} t_{\min } \geq B_{0} .
$$

Let us express an energy and momentum through the coordinate in a standard way:

$$
\varepsilon_{j}=\frac{p_{j}^{2}}{2}, \quad p_{j}=\frac{\Delta^{x_{j}}}{{ }_{\Delta} t_{\min }} .
$$

Then the energy increment ${ }_{\Delta} \varepsilon_{j}=p_{j} \cdot{ }_{\Delta} p_{j}$ can be substituted in the relation (44):

$$
{ }_{\Delta} x_{j} \cdot{ }_{\Delta} p_{j} \geq B_{0}
$$

Equations (44) and (45) connect uncertainties of the dynamic state definition. If we omit the "min" and " $j$ " indexes in the relation (44), these uncertainties may be represented in the following way:

$$
{ }_{\Delta} x \cdot{ }_{\Delta} p \geq B_{0}, \quad{ }_{\Delta} \varepsilon \cdot{ }_{\Delta} t \geq B_{0} .
$$

It was shown that a fixed boundary system has a discrete number of the phase states. Each of these states is characterized by its own phase diffusion $B_{j}$. In such a way the diffusive spectrum depends on the selected scale $k_{j}=(\pi$. $j) / L$. This property is well known for the case of a turbulent motion, when each scale corresponds to its own turbulent diffusion value [1]. In this case a standard molecular diffusion is only the minimal value for the molecular space scale. We have shown that for the considered case phase diffusion is the only parameter that defines a dynamic accuracy; that is why dynamic properties and a resolution in fact are scale dependent. Besides, according to the relation (32) small scales correspond to low diffusion and higher resolution abilities. Finally we come to the conclusion that the smaller scale we consider the more information we get about the motion.

\section{Conclusions}

In this paper uncertainties and clustering, lying in the basis of chaos, have been investigated. It was obtained that a chaotic system with a nonstationary diffusion satisfies a nonlinear dispersion law. This law leads to instabilities in a phase space and to the appearance of the clustering properties for the initially uniform system.

The generalized Fokker-Planck equation with a nonstationary diffusion has been derived. It has been applied to the analysis of a fixed boundary problem. An anomalous transport turned out to be a natural property of this equation under the considered conditions. This trivial law of diffusion led to a nontrivial output: relation of the coordinatemomentum uncertainties has been stated. We have shown 
that for the considered case phase diffusion is the only parameter that defines a dynamic accuracy; that is why dynamic properties and a resolution in fact are scale dependent.

Formulation of a generalized Fokker-Planck (GFP) equation, given in this paper, allows defining of an anomalous diffusion without complex fractal PDE. Data about the conservative spectrum of GFP may help to find stable states of real complex systems and avoid their destruction. A clustering formal explanation helps to define and anticipate attraction areas in the real phase spaces. At the same time derived uncertainty relations allow us to understand limits of the possible forecast efficiency through the connection of the accuracy with transport properties. The author believes that represented results can be used in the area of complex systems modelling and forecast.

\section{Conflict of Interests}

The author declares that there is no conflict of interests regarding the publication of this paper.

\section{Acknowledgments}

The author would like to thank revisers of Journal of Chaos for a careful study of the current paper and detailed critical comments. The author would like to express his deep gratitude to the editor Dr. Grzegorz Litak for his valuable remarks.

\section{References}

[1] L. D. Landau and E. M. Lifshitz, Hydrodynamics, Fismatlit, Moscow, Russia, 2007.

[2] E. Lorentz, Deterministic Nonperiodic Motion, Strange Attractors, Moscow, Russia, 1981.

[3] M. J. Feigenbaum, "The universal metric properties of nonlinear transformations," Journal of Statistical Physics, vol. 21, no. 6, pp. 669-706, 1979.

[4] J. Moser, "On invariant curves of area preserving mappings on an annulus," in Nachrichten der Akademie der Wissenschaften in Göttingen, Mathematisch-Physikalische, vol. 1, pp. 1-20, 1962.

[5] G. M. Zaslavsky and R. Z. Sagdeev, Introduction to Nonlinear Physics: From the Pendulum to Turbulence and Chaos, Nauka, Moscow, Russia, 1988.

[6] G. M. Zaslavsky, The Physics of Chaos in Hamiltonian Systems, Imperial College Press, London, UK, 2007. 


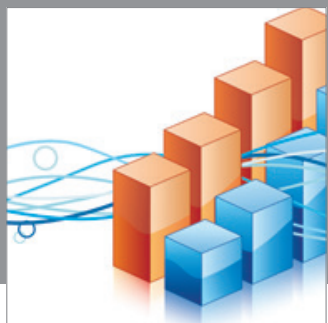

Advances in

Operations Research

mansans

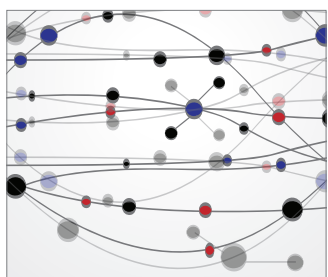

The Scientific World Journal
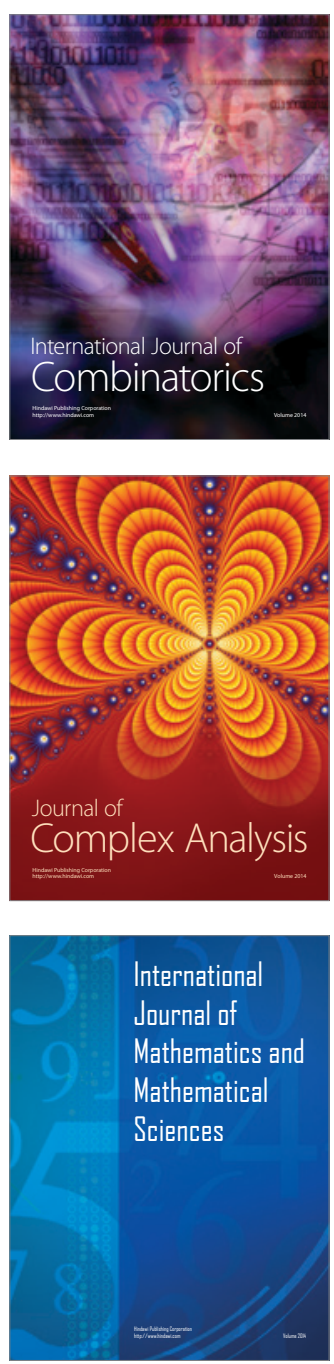
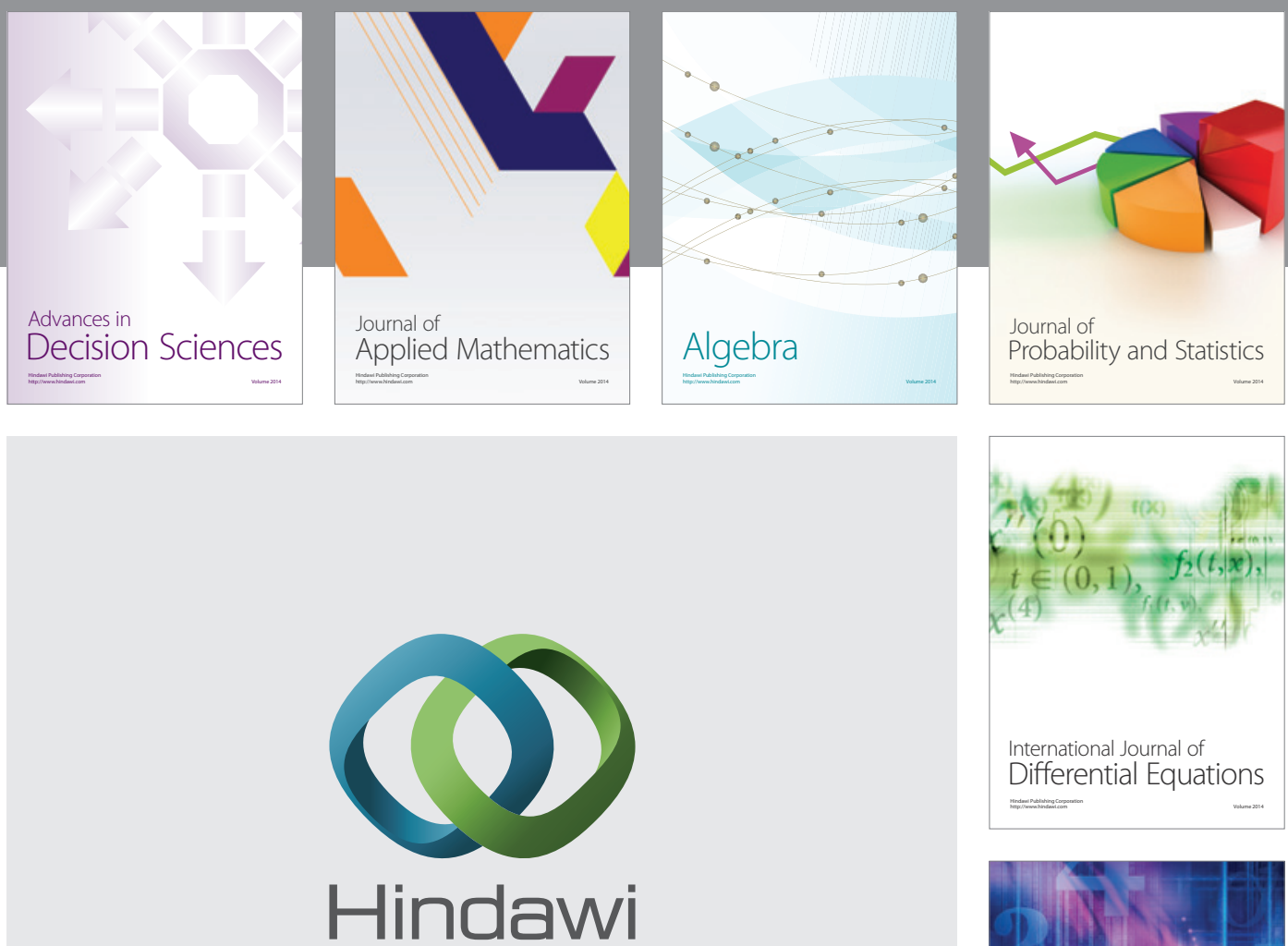

Submit your manuscripts at http://www.hindawi.com
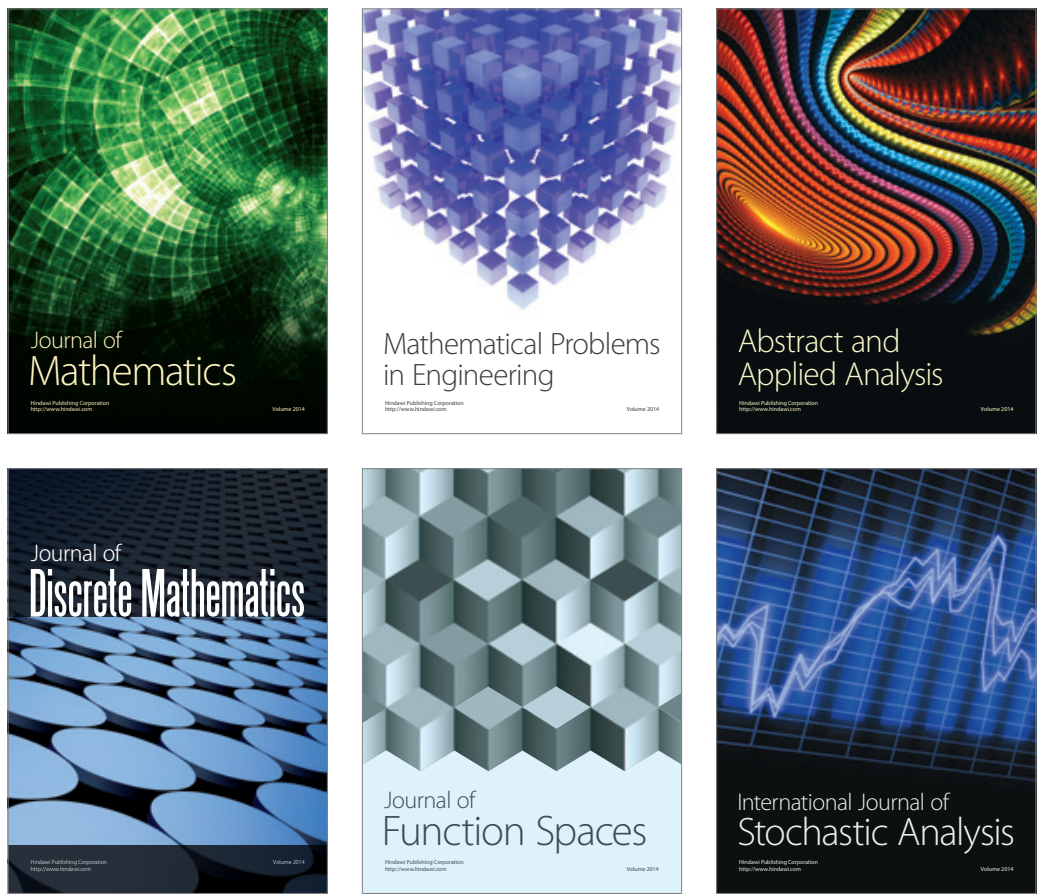

Journal of

Function Spaces

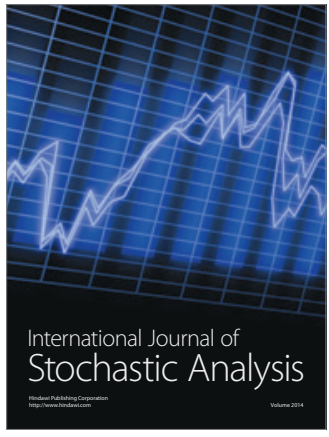

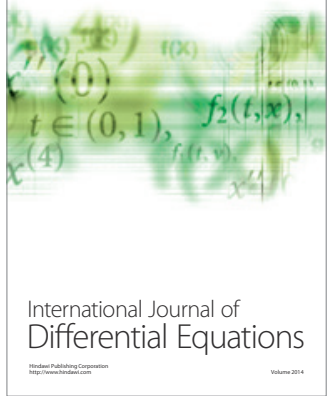
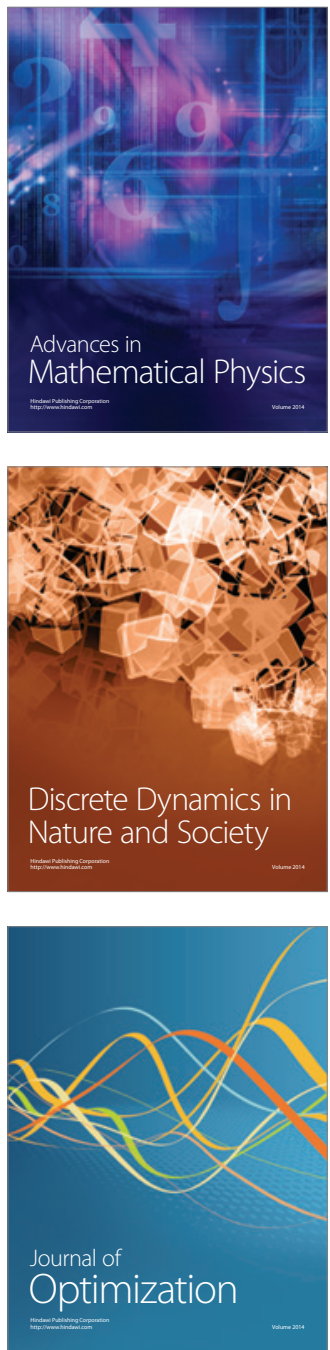